\title{
Influence of Water Regimes and Potassium Chlorate on Floral Induction, Leaf Photosynthesis and Leaf Water Potential in Longan
}

\author{
Chiti Sritontip ${ }^{1,2,5}$, Pimsiri Tiyayon ${ }^{3}$, Korawan Sringam ${ }^{4}$, Sanchai Pantachod ${ }^{1}$, Darunee Naphrom ${ }^{5}$, \\ Soraya Ruamrungsri ${ }^{5}$ \& Pittaya Sruamsiri ${ }^{2,5}$ \\ ${ }^{1}$ Agricultural Technology Research Institute, Rajamangala University of Technology Lanna, Lampang, Thailand \\ ${ }^{2}$ Central of Excellence on Agricultural Biotechnology (AG-BIO/PERDO-CHE), Bangkok, Thailand \\ ${ }^{3}$ The Office of the Commission on Agricultural Resource Education, Chulalongkorn University, Bangkok, \\ Thailand \\ ${ }^{4}$ Central Laboratory, Faculty of Agriculture, Chiang Mai University, Chiang Mai, Thailand \\ ${ }^{5}$ Department of Plant Science and Natural Resources, Faculty of Agriculture, Chiang Mai University, Chiang Mai, \\ Thailand \\ Correspondence: Chiti Sritontip, Agricultural Technology Research Institute, Rajamangala University of \\ Technology Lanna, Lampang, Thailand. Tel: 66-054-342-553.E-mail: Chiti_s@hotmail.com
}

Received: February 18, 2013 Accepted: April 2, 2013 Online Published: May 15, 2013

doi:10.5539/jas.v5n6p211

URL: http://dx.doi.org/10.5539/jas.v5n6p211

\begin{abstract}
This study verifies the influence of water regimes and potassium chlorate $\left(\mathrm{KClO}_{3}\right)$ on photosynthetic rate, flower emergence and media moisture content of longan trees. The trees were grown in 150 liters lysimeter tanks filled with fine sand. The experimental design was a $2 \times 2$ factorial in completely randomized design (CRD) with 2 factors; 1) two levels of water regimes (well-watered and water deficit) and 2) two levels of $\mathrm{KClO}_{3}$ at 10 and $0 \mathrm{~g}$. The results revealed that the well-watered treatment produced faster days of terminal bud break than that of the water deficit treatment. The $10 \mathrm{~g} \mathrm{KClO}_{3}$ treatment induced $91 \%$ flower emergence at 35 days after commencing the treatment, while the $0 \mathrm{~g} \mathrm{KClO}_{3}$ treatment had $82 \%$ leaf flushing and had no flower emergence. Water deficit or $\mathrm{KClO}_{3}$ treatments reduced the net carbondioxide $\left(\mathrm{CO}_{2}\right)$ exchange, transpiration and stomatal conductance rates. Moreover, the combination of well-watered and $0 \mathrm{~g} \mathrm{KClO}_{3}$ treatments gave the greatest values of the parameters. The well-watered treatment had higher volumetric water content in the growing medium and leaf water potential than the water deficit treatment, while for the $10 \mathrm{~g}$ and $0 \mathrm{~g} \mathrm{KClO}_{3}$ treatments had similar the media moisture content.
\end{abstract}

Keywords: potassium chlorate, water deficit, the net $\mathrm{CO}_{2}$ exchange rate

\section{Introduction}

Longan (Dimocarpus longan Lour.) is one of the most popular fruit crops of northern Thailand. Longan flowers from late December to late February and is harvested from late June to August. At the present, the main region of longan cultivation is in the upper northern part of the country, such as 'Daw', 'Haeo', 'Bieo Khieo' and 'Si Chomphu'. Floral induction is an important step of flowering and fruiting. Due to the fact that the soil moisture levels and levels of $\mathrm{KClO}_{3}$ used vary from place to place, induction of flowering at certain times is necessary in longan production. There are many factors that control flowering in longan, such as temperature, tree health, cultivar, water stress and potassium chlorate. (Subhadrabandhu, 1990; Manochai et al., 2004; Davenport \& Stern, 2005; Sritontip et al., 2005).

Water constitutes a major part of the tissue mass and is required for growth and development. Plant water status is a good indicator of plant health and how well adapted the plant is to its environment. Plant water status can provide information on potential crop yield or be used for irrigation strategy. The water potential of a plant governs transport across cell membranes. Water potential can be used to evaluate the water status of a plant and provides a relative index of water stress. Technological advancements have increased the relative ease and number of variations to measure water potential in plant or plant leaves. Water is the most important factor among the environmental factors affecting growth; it may reduce the growth rate, metabolic activities and leaf area 
(Metheneg et al., 1994). Soil water content should be correlated with physiological responses; growth and fruit production in fruit crops, to determine the appropriate amount of water to apply to the crop. Irrigation intervals and water supply for the vegetative growth reduction have counter relationships (Blum, 1996). However, a few studies have indicated that water limitation can have economically beneficial consequences on fruit production (Caspari et al., 1994; Chalmers et al., 1981; Shackel et al., 2000). In Asian pear, water stress reduces vegetative growth but increases return bloom and decreases flesh to dry weight ratio (Caspari et al., 1994; Shackel et al., 2000). A water deficit condition reduces plant growth and affects photosynthesis and that, in turn, reduces leaf area, enhances stomata closure, decreases water status in the leaf tissue, reduces the rate of $\mathrm{CO}_{2}$ assimilated, causes ultra structural changes in chloroplasts, affects electron transport and $\mathrm{CO}_{2}$ assimilation reaction and alters the level of photosynthesis in tissues (Dubey, 1997; Fitter, 1987). In 1998, Thai longan growers have begun to apply potassium chlorate $\left(\mathrm{KClO}_{3}\right)$ to induce off-season flowering, the methods applied potassium chlorate by soil drench, foliar spray and stem injection (Sritontip et al., 2005; Davenport \& Stern, 2005; Manochai et al., 2005). However, the Chlorate group chemical is a strong oxidizing agent and considered phytotoxic to all the green parts of plant cell (Stecher et al., 1960; Thomson, 1993). The $\mathrm{KClO}_{3}$ is dissociated into potassium ion $\left(\mathrm{K}^{+}\right)$and chlorate ion $\left(\mathrm{ClO}_{3}{ }^{-}\right)$when dissolves in water. Chlorate or chlorine is chemically analog of nitrate $\left(\mathrm{NO}_{3}{ }^{-}\right)$and used widely as a herbicide. The reduction products, chlorite $\left(\mathrm{ClO}_{2}^{-}\right)$and hypochlorite $\left(\mathrm{ClO}^{-}\right)$have been shown to be rapidly acting toxins that poisoned all cell types. Root growth is severally inhibited and leaf is yellow, withered and die (LaBrie et al., 1991). It was previously shown that the low temperature and $\mathrm{KClO}_{3}$ applications decreased leaf photosynthesis in longan tree (Sritontip et al., 2010). Although, $\mathrm{KClO}_{3}$ has been extensively used to promote flowering in longan production, the mode of action and mechanism of flower induction is not yet clear. However, the effect of water deficit and/or $\mathrm{KClO}_{3}$ on flower induction of longan is still lacking. Thus, this research studies what effects water deficit and $\left(\mathrm{KClO}_{3}\right)$ applications have on leaf photosynthesis change and flower induction in longan.

\section{Method}

\subsection{Plant Material and Lysimeter Tank Facility}

The sixteen of two-years-old air layered longan trees cv. Daw were grown in lysimeters with a capacity of 150 liters consisting of special containers filled with sand and connected by a tube to a 30 liter plastic container with nutrient solution as indicated in Figure 1.

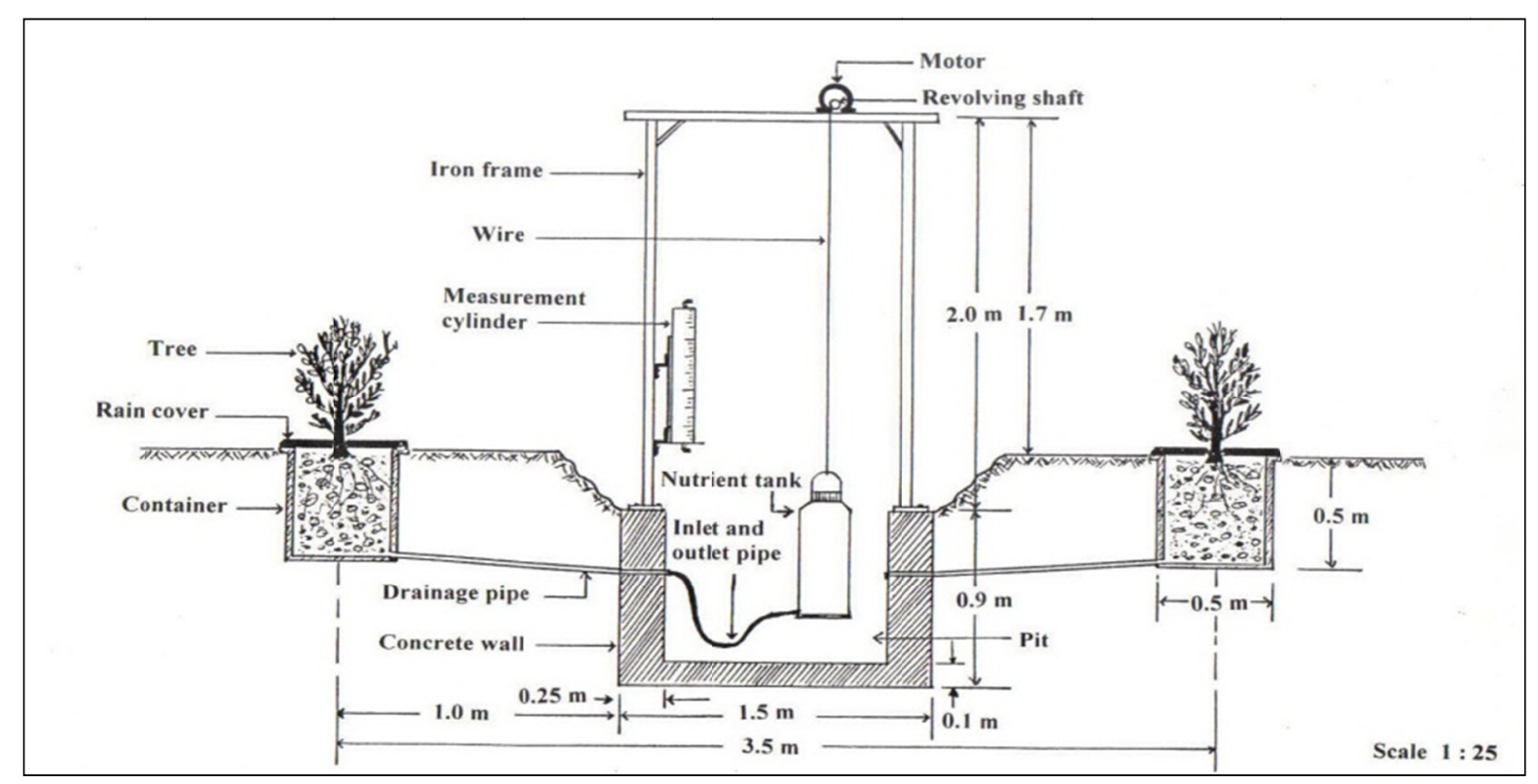

Figure 1. Schematic cross-section diagram of the lysimeter facility

A lysimeter facility was built at the Agricultural Technology Research Institute, Rajamagala University of Technology Lanna, Lampang in 1999 to study the water relationships of fruit crops. For precise measurement of water use by fruit crops in a lysimeter the soil-water status can be controlled more accurately than in the field. The ion concentration of the applied nutrient solution is indicated in Tab 1. 
Table 1. Composition of the standard nutrient solution* used for longan trees growing in lysimeters

\begin{tabular}{llll}
\hline Cations & meq/l & Anion & meq/l \\
\hline $\mathrm{Ca}^{2+}$ & 7.0 & $\mathrm{NO}_{3}^{-}$ & 7.0 \\
$\mathrm{~K}^{+}$ & 4.0 & $\mathrm{PO}_{4}-$ & 3.0 \\
$\mathrm{Mg}^{2+}$ & 4.0 & $\mathrm{SO}_{4}^{-}$ & 8.0 \\
$\mathrm{NH}_{4}^{+}$ & 1.0 & & \\
$\mathrm{H}^{+}$ & 2.0 & & \\
\hline Total & 18.0 & Total & 18.0
\end{tabular}

*Micronutrients were added according to [11], the nutrient solution $\mathrm{pH}$ was adjusted weekly to 6.5 by addition of $\mathrm{H}_{2} \mathrm{SO}_{4}$.

\subsection{Experiment Treatment}

The experimental design was a $2 \times 2$ factorial in completely randomized design (CRD) with 4 replications, total of 16 longan tree pots, with water regimes of well-watered (WW) or water deficit (WD) and $\mathrm{KClO}_{3}$ at 10 and $0 \mathrm{~g}$. pot $^{-1}$. The WW treated plants were supplied daily with a constant volume of water of 30 liters throughout the experimental period. The WD treated plants received a starting amount of water of 15 liters (in the 30-liter capacity container) and were supplied daily until the water container was empty. Then, the container was refilled to the starting volume of 15 liters. The nutrient solution was replaced every 15 day. The longan trees at the fully mature leaf stage were treated with $10 \mathrm{~g} \mathrm{pot}^{-1} \mathrm{KClO}_{3}$ mixed into the nutrient solution containers. The study was conducted in the lysimeters at the Agricultural Technology Research Institute, Rajamangala University of Technology Lanna, Lampang, Thailand from November 1, 2008 to January 31, 2009.

\subsection{Data Collection}

1. Percentage of flowering, the sixteenth longan trees were sampled for data. These data were the percentage and days to visible active buds. The data collection lasted 49 days after treatment.

2. Leaf photosynthesis and chlorophyll fluorescence were measured immediately after $\mathrm{KClO}_{3}$ application and then monitored at 1, 4, 7, 10,13,17,21, 28 and 35 days after $\mathrm{KClO}_{3}$ application (4 leaves per tree were sampled). The measurements were made on the $3^{\text {rd }}$ or $4^{\text {th }}$ leaf position of the fully expanded mature compound leaves at 09.00 to 10.00 a.m. Chlorophyll fluorescence $(\mathrm{Fv} / \mathrm{Fm})$ was measured at the leaf using a plant efficiency analyzer (Model PEA, Hansatech Instruments, UK.). On the same leaf, net $\mathrm{CO}_{2}$ exchange, transpiration, and stomatal conductance rates were measured using a portable steady-state leaf photosynthesis system at an irradiance of 1,200 $\mu \mathrm{mol} \mathrm{m}^{-2}$ $\mathrm{s}^{-1}$ PAR (Model LCA-4 with the PLC-4 leaf chamber; Analytical Development Company Ltd. (ADC), UK).

3. The moisture content in each growing media was measured with a moisture meter (Type HH2, Delta-T Devices Ltd., UK) and profile probe (Type PR2-UM-2.0, Delta-T Devices Ltd., UK) at 1, 4, 7, 10, 13, 17, 21, 28 and 35 days after $\mathrm{KClO}_{3}$ application.

4. Longan trees were measured for leaf water potential by a pressure bomb (Plant Water Status Console-Model 3005, Soilmoisture Equipment Corp, USA) ) at 1, 4, 7, 10, 13, 17, 21, 28 and 35 days after $\mathrm{KClO}_{3}$ application.

\subsection{Data Analysis}

The data was analyzed for statistical significance by using the Statistic 8 analytical software package (SXW Tallahassee, FL). The Least Significant Difference (LSD) was used to compare treatment differences with ANOVA $(\mathrm{P}<0.05)$.

\section{Results}

\subsection{The Effects of Water Regimes and $\mathrm{KClO}_{3}$ on the Physiology of the Trees}

The longan trees that were treated with well watered produced terminal bud break significantly earlier than that of water deficit. Different water regimes had no differences on percentage of floral emergence or leaf flushing measurements after commencement of treatment. Those treated with $10 \mathrm{~g} \mathrm{KClO}_{3}$ had greater floral emergence and lower leaf flushing. However, the $10 \mathrm{~g}$ and $0 \mathrm{~g} \mathrm{KClO}_{3}$ were similar in the days of terminal bud break. There was no interaction between the water regimes and $\mathrm{KClO}_{3}$ treatments on days of terminal bud break, percentage of flower emergence or leaf flushing after treatment (Table 2). 
Table 2. Changes in terminal bud break, floral emergence and leaf flushing after start of the treatments

\begin{tabular}{llll}
\hline Factors & $\begin{array}{l}\text { Days of terminal bud } \\
\text { break }\end{array}$ & $\begin{array}{l}\text { Percentage of floral emergence after } \\
\text { treatment }\end{array}$ & $\begin{array}{l}\text { Percentage of leaf flushing after } \\
\text { treatment }\end{array}$ \\
\hline Water & & & \\
$(\mathrm{A})$ & & 47.82 & 45.89 \\
$\mathrm{WW}$ & $26 \mathrm{~b}$ & 43.34 & 44.95 \\
$\mathrm{WD}$ & $35 \mathrm{a}$ & & \\
$\mathrm{KClO}$ & & & $8.84 \mathrm{~b}$ \\
$(\mathrm{~B})$ & & $91.00 \mathrm{a}$ & $82.00 \mathrm{a}$ \\
$10 \mathrm{~g}$ & 32 & $0 \mathrm{~b}$ & $\mathrm{NS}$ \\
$0 \mathrm{~g}$. & 29 & $\mathrm{NS}$ & $*$ \\
\hline $\mathrm{A}$ & $*$ & $*$ & $\mathrm{NS}$ \\
$\mathrm{B}$ & $\mathrm{NS}$ & $\mathrm{NS}$ & \\
$\mathrm{A} x \mathrm{~B}$ & $\mathrm{NS}$ & $\mathrm{n}$ & \\
\hline
\end{tabular}

*Means within the column followed by the same letter were not significantly different at $\mathrm{p}=0.05$ by LSD. NS $=$ Non-significant.

\subsection{Changes in the Leaf Photosynthesis Characteristics Caused by Water Regimes and $\mathrm{KClO}_{3}$}

The water deficit longan trees had efficiency of photosystem II lower at 7, 10, and 28 days after commencement treatment. Moreover, the $10 \mathrm{~g} \mathrm{KClO}_{3}$ reduced the efficiency of photosystem II at 4, 7, 10 and 28 days after application (Table 3). The interaction effect showed that the well water with $0 \mathrm{~g} \mathrm{KClO}$, had the highest value for the efficiency of photosystem II (Table 4).

Table 3. Chlorophyll fluorescence $(\mathrm{Fv} / \mathrm{Fm})$ changes of 'Daw' longan trees after $\mathrm{KClO}_{3}$ and water regime treatments

\begin{tabular}{lllllllllll}
\hline \multirow{2}{*}{ Factors } & \multicolumn{10}{c}{ Time after application (days) } \\
\cline { 2 - 11 } & 1 & 4 & 7 & 10 & 13 & 17 & 21 & 28 & 35 \\
\hline Water (A) & & & & & & & & & & \\
$\mathrm{WW}$ & 0.69 & 0.70 & $0.70 \mathrm{a}$ & $0.68 \mathrm{a}$ & 0.63 & 0.69 & 0.69 & $0.65 \mathrm{a}$ & 0.62 \\
$\mathrm{WD}$ & 0.66 & 0.66 & $0.61 \mathrm{~b}$ & $0.58 \mathrm{~b}$ & 0.59 & 0.63 & 0.64 & $0.56 \mathrm{~b}$ & 0.54 \\
\hline $\mathrm{KClO}_{3}(\mathrm{~B})$ & & & & & & & & & & \\
$10 \mathrm{~g}$ & 0.66 & $0.64 \mathrm{~b}$ & $0.61 \mathrm{~b}$ & $0.59 \mathrm{~b}$ & 0.58 & 0.64 & 0.64 & $0.56 \mathrm{~b}$ & 0.55 \\
$0 \mathrm{~g}$. & 0.69 & $0.72 \mathrm{a}$ & $0.70 \mathrm{a}$ & $0.67 \mathrm{a}$ & 0.64 & 0.68 & 0.69 & $0.64 \mathrm{a}$ & 0.61 \\
\hline $\mathrm{A}$ & $\mathrm{NS}$ & $\mathrm{NS}$ & $*$ & $*$ & $\mathrm{NS}$ & $\mathrm{NS}$ & $\mathrm{NS}$ & $*$ & $*$ \\
$\mathrm{~B}$ & $\mathrm{NS}$ & $*$ & $*$ & $*$ & $\mathrm{NS}$ & $\mathrm{NS}$ & $\mathrm{NS}$ & $*$ & $\mathrm{NS}$ \\
$\mathrm{Ax} \mathrm{B}$ & $\mathrm{NS}$ & $\mathrm{NS}$ & $*$ & $*$ & $\mathrm{NS}$ & $*$ & $*$ & $*$ & $*$ \\
\hline
\end{tabular}

*Means within the column followed by the same letter were not significantly different at $\mathrm{p}=0.05$ by LSD. NS $=$ Non-significant.

Table 4. Interaction effect of $\mathrm{KClO}_{3}$ and water regime on chlorophyll fluorescence ( $\left.\mathrm{Fv} / \mathrm{Fm}\right)$ of 'Daw' longan trees after treatment

\begin{tabular}{llllllllll}
\hline \multirow{2}{*}{ Treatments } & \multicolumn{8}{c}{ Time after application (days) } \\
\cline { 2 - 11 } & 1 & 4 & 7 & 10 & 13 & 17 & 21 & 28 & 35 \\
\hline $\mathrm{WW}+\mathrm{KClO}_{3}$ & 0.66 & 0.66 & $0.64 \mathrm{ab}$ & $0.62 \mathrm{~b}$ & 0.60 & $0.65 \mathrm{ab}$ & $0.68 \mathrm{ab}$ & $0.60 \mathrm{~b}$ & $0.60 \mathrm{ab}$ \\
$\mathrm{WW}^{-\mathrm{KClO}_{3}}$ & 0.71 & 0.75 & $0.76 \mathrm{a}$ & $0.74 \mathrm{a}$ & 0.66 & $0.72 \mathrm{a}$ & $0.71 \mathrm{a}$ & $0.69 \mathrm{a}$ & $0.65 \mathrm{a}$ \\
$\mathrm{WD}+\mathrm{KClO}_{3}$ & 0.65 & 0.62 & $0.57 \mathrm{~b}$ & $0.55 \mathrm{~b}$ & 0.56 & $0.62 \mathrm{~b}$ & $0.61 \mathrm{~b}$ & $0.52 \mathrm{~b}$ & $0.51 \mathrm{~b}$ \\
$\mathrm{WD}-\mathrm{KClO}_{3}$ & 0.67 & 0.69 & $0.65 \mathrm{ab}$ & $0.60 \mathrm{~b}$ & 0.62 & $0.64 \mathrm{ab}$ & $0.67 \mathrm{ab}$ & $0.59 \mathrm{~b}$ & $0.57 \mathrm{a}$ \\
\hline F-test & $\mathrm{NS}$ & $\mathrm{NS}$ & $*$ & $*$ & $\mathrm{NS}$ & $*$ & $*$ & $*$ & $*$ \\
\hline
\end{tabular}

*Means within the column followed by the same letter were not significantly different at $\mathrm{p}=0.05$ by LSD. NS $=$ Non-significant. 
The water deficit treatments decreased the net $\mathrm{CO}_{2}$ exchange rate during 1 to 35 days after treatment and the $10 \mathrm{~g}$ $\mathrm{KClO}_{3}$ had a net $\mathrm{CO}_{2}$ exchange rate that was lower than the $0 \mathrm{~g} \mathrm{KClO}_{3}$ at $4,7,10,13$, and 21 days after treatment (Table 5). The interaction effect between water regimes and $\mathrm{KClO}_{3}$ rates showed that the well water with $0 \mathrm{~g}$ $\mathrm{KClO}_{3}$ had the greatest effect on the net $\mathrm{CO}_{2}$ exchange rate of those treatments (Table 6).

Table 5. Effects of water regimes and $\mathrm{KClO}_{3}$ rates on the net $\mathrm{CO}_{2}$ exchange rate $\left(\mu \mathrm{mol} \mathrm{M}^{-2} \mathrm{~S}^{-1}\right)$ after treatment

\begin{tabular}{llllllllll}
\hline \multirow{2}{*}{ Factors } & \multicolumn{8}{c}{ Time after application (days) } \\
\cline { 2 - 10 } & 1 & 4 & 7 & 10 & 13 & 17 & 21 & 28 & 35 \\
\hline Water (A) & & & & & & & & & \\
$\mathrm{WW}$ & $5.58 \mathrm{a}$ & $3.95 \mathrm{a}$ & $3.90 \mathrm{a}$ & $5.12 \mathrm{a}$ & 4.75 & $7.36 \mathrm{a}$ & $5.61 \mathrm{a}$ & $4.12 \mathrm{a}$ & $4.84 \mathrm{a}$ \\
$\mathrm{WD}$ & $2.99 \mathrm{~b}$ & $2.62 \mathrm{~b}$ & $2.05 \mathrm{~b}$ & $2.68 \mathrm{~b}$ & 1.91 & $3.71 \mathrm{~b}$ & $3.96 \mathrm{~b}$ & $2.80 \mathrm{~b}$ & $3.08 \mathrm{~b}$ \\
$\mathrm{KClO}_{3}(\mathrm{~B})$ & & & & & & & & & \\
$10 \mathrm{~g}$ & 3.87 & $2.17 \mathrm{~b}$ & $1.89 \mathrm{~b}$ & $2.20 \mathrm{~b}$ & $2.26 \mathrm{~b}$ & 4.32 & $3.82 \mathrm{~b}$ & 3.06 & 3.67 \\
$0 \mathrm{~g}$. & 4.70 & $4.40 \mathrm{a}$ & $4.07 \mathrm{a}$ & $5.60 \mathrm{a}$ & $4.41 \mathrm{a}$ & 6.75 & $5.75 \mathrm{a}$ & 3.85 & 4.25 \\
\hline $\mathrm{A}$ & $*$ & $*$ & $*$ & $*$ & $*$ & $*$ & $*$ & $*$ & $*$ \\
$\mathrm{~B}$ & $\mathrm{NS}$ & $*$ & $*$ & $*$ & $*$ & $\mathrm{NS}$ & $*$ & $\mathrm{NS}$ & $\mathrm{NS}$ \\
$\mathrm{A} \times \mathrm{B}$ & $\mathrm{NS}$ & $*$ & $*$ & $*$ & $*$ & $*$ & $\mathrm{NS}$ & $\mathrm{NS}$ & $\mathrm{NS}$
\end{tabular}

* Means within the column followed by the same letter were not significantly different at $\mathrm{p}=0.05$ by LSD. NS $=$ Non-significant.

Table 6. Interaction effect of water regimes and $\mathrm{KClO}_{3}$ rates on the net $\mathrm{CO}_{2}$ exchange rate $\left(\mu\right.$ mol M $\left.\mathrm{M}^{-2} \mathrm{~S}^{-1}\right)$ after treatment

\begin{tabular}{llllllllll}
\hline \multirow{2}{*}{ Treatments } & \multicolumn{8}{c}{ Time after application (days) } \\
\cline { 2 - 11 } & 1 & 4 & 7 & 10 & 13 & 17 & 21 & 28 & 35 \\
\hline $\mathrm{WW}+\mathrm{KClO}_{3}$ & 4.81 & $2.10 \mathrm{~b}$ & $2.43 \mathrm{~b}$ & $3.06 \mathrm{~b}$ & $3.90 \mathrm{~b}$ & $5.63 \mathrm{ab}$ & 4.40 & 3.83 & 4.40 \\
$\mathrm{WW}-\mathrm{KClO}_{3}$ & 6.34 & $5.80 \mathrm{a}$ & $5.38 \mathrm{a}$ & $7.18 \mathrm{a}$ & $5.60 \mathrm{a}$ & $9.08 \mathrm{a}$ & 6.82 & 4.40 & 5.28 \\
$\mathrm{WD}+\mathrm{KClO}_{3}$ & 2.94 & $2.24 \mathrm{~b}$ & $1.36 \mathrm{c}$ & $1.34 \mathrm{~b}$ & $0.62 \mathrm{c}$ & $3.00 \mathrm{~b}$ & 3.25 & 2.30 & 2.94 \\
$\mathrm{WD}-\mathrm{KClO}_{3}$ & 3.05 & $3.00 \mathrm{~b}$ & $2.75 \mathrm{~b}$ & $4.01 \mathrm{~b}$ & $3.21 \mathrm{~b}$ & $4.41 \mathrm{~b}$ & 4.68 & 3.30 & 3.23 \\
\hline F-test & $\mathrm{NS}$ & $*$ & $*$ & $*$ & $*$ & $*$ & NS & NS & NS
\end{tabular}

* Means within the column followed by the same letter were not significantly different at $\mathrm{p}=0.05$ by LSD. NS $=$ Non-significant.

For changes in transpiration rate, the water deficit had lower transpiration rate than the well-watered at 7 and 10 days after treatment and the $10 \mathrm{~g} \mathrm{KClO}_{3}$ treatment depressed the transpiration rate at 4-17 days after treatment (Table 7). The interaction effect between water regimes and $\mathrm{KClO}_{3}$ resulted that the well-watered with $10 \mathrm{~g} \mathrm{KClO}$, the water deficit with $10 \mathrm{~g} \mathrm{KClO}_{3}$ and water deficit with $0 \mathrm{~g} \mathrm{KClO}_{3}$ treatments decreased the transpiration rate when compared with the well-watered with $0 \mathrm{~g} \mathrm{KClO}_{3}$ treatment (Table 8).

Table 7. Effects of water regimes and $\mathrm{KClO}_{3}$ rates on the transpiration rate $\left(\mathrm{m} \mathrm{mol} \mathrm{M}^{-2} \mathrm{~S}^{-1}\right)$ after treatment

\begin{tabular}{lllllllllll}
\hline \multirow{2}{*}{ Factors } & \multicolumn{10}{c}{ Time after application (days) } \\
\cline { 2 - 11 } & 1 & 4 & 7 & 10 & 13 & 17 & 21 & 28 & 35 \\
\hline Water (A) & & & & & & & & & & \\
$\mathrm{WW}$ & 1.09 & 1.08 & $0.78 \mathrm{a}$ & $1.24 \mathrm{a}$ & 1.07 & 0.97 & 0.98 & 0.86 & 1.04 \\
$\mathrm{WD}$ & 0.95 & 0.82 & $0.43 \mathrm{~b}$ & $0.90 \mathrm{~b}$ & 0.97 & 0.82 & 0.99 & 0.63 & 0.95 \\
$\mathrm{KClO}_{3}(\mathrm{~B})$ & & & & & & & & & & \\
$10 \mathrm{~g}$ & 0.92 & $0.79 \mathrm{~b}$ & $0.47 \mathrm{~b}$ & $0.75 \mathrm{~b}$ & $0.84 \mathrm{~b}$ & $0.65 \mathrm{~b}$ & 0.90 & 0.66 & 0.93 \\
$0 \mathrm{~g}$. & 1.10 & $1.08 \mathrm{a}$ & $0.72 \mathrm{a}$ & $1.33 \mathrm{a}$ & $1.16 \mathrm{a}$ & $1.08 \mathrm{a}$ & 1.05 & 0.82 & 1.04 \\
\hline $\mathrm{A}$ & $\mathrm{NS}$ & $\mathrm{NS}$ & $*$ & $*$ & $\mathrm{NS}$ & $\mathrm{NS}$ & $\mathrm{NS}$ & $\mathrm{NS}$ & $\mathrm{NS}$ \\
$\mathrm{B}$ & $\mathrm{NS}$ & $*$ & $*$ & $*$ & $*$ & $*$ & $\mathrm{NS}$ & $\mathrm{NS}$ & $\mathrm{NS}$ \\
$\mathrm{A} \times \mathrm{B}$ & $\mathrm{NS}$ & $\mathrm{NS}$ & $*$ & $*$ & $*$ & $*$ & $\mathrm{NS}$ & $*$ & $\mathrm{NS}$ \\
\hline
\end{tabular}

* Means within the column followed by the same letter were not significantly different at $\mathrm{p}=0.05$ by LSD. NS $=$ Non-significant. 
Table 8. Interaction effect of water regimes and $\mathrm{KClO}_{3}$ rates on the transpiration rate $\left(\mathrm{m} \mathrm{mol} \mathrm{M}^{-2} \mathrm{~S}^{-1}\right)$ after treatment

\begin{tabular}{llllllllll}
\hline \multirow{2}{*}{ Treatments } & \multicolumn{7}{c}{ Time after application (days) } \\
\cline { 2 - 10 } & 1 & 4 & 7 & 10 & 13 & 17 & 21 & 28 & 35 \\
\hline $\mathrm{WW}+\mathrm{KClO}_{3}$ & 1.02 & 0.90 & $0.54 \mathrm{~b}$ & $0.81 \mathrm{ab}$ & $0.89 \mathrm{~b}$ & $0.72 \mathrm{~b}$ & 0.88 & $0.62 \mathrm{~b}$ & 0.94 \\
$\mathrm{WW}-\mathrm{KClO}_{3}$ & 1.17 & 1.26 & $1.02 \mathrm{a}$ & $1.67 \mathrm{a}$ & $1.26 \mathrm{a}$ & $1.22 \mathrm{a}$ & 1.08 & $1.10 \mathrm{a}$ & 1.15 \\
$\mathrm{WD}+\mathrm{KClO}_{3}$ & 0.82 & 0.67 & $0.39 \mathrm{~b}$ & $0.68 \mathrm{c}$ & $0.79 \mathrm{~b}$ & $0.59 \mathrm{~b}$ & 0.93 & $0.70 \mathrm{~b}$ & 0.93 \\
$\mathrm{WD}-\mathrm{KClO}_{3}$ & 1.09 & 0.97 & $0.48 \mathrm{~b}$ & $1.12 \mathrm{ab}$ & $1.14 \mathrm{ab}$ & $1.04 \mathrm{a}$ & 1.06 & $0.57 \mathrm{~b}$ & 0.97 \\
\hline F-test & $\mathrm{NS}$ & $\mathrm{NS}$ & $*$ & $*$ & $*$ & $*$ & $\mathrm{NS}$ & $*$ & $\mathrm{NS}$
\end{tabular}

*Means within the column followed by the same letter were not significantly different at $\mathrm{p}=0.05$ by LSD. NS $=$ Non-significant.

The water deficit reduced the stomatal conductance at 7, 13, 17 and 28 days after treatment. A similar result was obtained with the $10 \mathrm{~g} \mathrm{KClO}_{3}$ where the stomatal conductance declined at 4, 7, 10, 13, 17 and 28 days after treatment (Table 9). The interaction effect between water regimes and $\mathrm{KClO}_{3}$ rates showed that the well-watered with $0 \mathrm{~g} \mathrm{KClO}_{3}$ treatment had the greatest stomatal conductance after the beginning of the experiment (Table 10).

Table 9. Effects of water regimes and $\mathrm{KClO}_{3}$ rates on the stomatal conductance rate $\left(\mathrm{mol} \mathrm{M}^{-2} \mathrm{~S}^{-1}\right)$ after treatment

\begin{tabular}{lllllllllll}
\hline \multirow{2}{*}{ Factors } & \multicolumn{8}{c}{ Time after application (days) } \\
\cline { 2 - 10 } & 1 & 4 & 7 & 10 & 13 & 17 & 21 & 28 & 35 \\
\hline Water (A) & & & & & & & & & \\
WW & 0.06 & 0.04 & $0.05 \mathrm{a}$ & $0.05 \mathrm{a}$ & $0.05 \mathrm{a}$ & 0.05 & 0.06 & $0.03 \mathrm{a}$ & 0.05 \\
$\mathrm{WD}$ & 0.04 & 0.03 & $0.02 \mathrm{~b}$ & $0.03 \mathrm{~b}$ & $0.04 \mathrm{~b}$ & 0.04 & 0.06 & $0.02 \mathrm{~b}$ & 0.05 \\
$\mathrm{KClO}_{3}(\mathrm{~B})$ & & & & & & & & & \\
$10 \mathrm{~g}$ & 0.04 & $0.02 \mathrm{~b}$ & $0.02 \mathrm{~b}$ & $0.03 \mathrm{~b}$ & $0.03 \mathrm{~b}$ & $0.04 \mathrm{~b}$ & 0.05 & $0.02 \mathrm{~b}$ & 0.05 \\
$0 \mathrm{~g}$. & 0.06 & $0.04 \mathrm{a}$ & $0.04 \mathrm{a}$ & $0.05 \mathrm{a}$ & $0.05 \mathrm{a}$ & $0.06 \mathrm{a}$ & 0.06 & $0.03 \mathrm{a}$ & 0.05 \\
\hline $\mathrm{A}$ & $\mathrm{NS}$ & $\mathrm{NS}$ & $*$ & $*$ & $*$ & $\mathrm{NS}$ & $\mathrm{NS}$ & $*$ & $\mathrm{NS}$ \\
$\mathrm{B}$ & $\mathrm{NS}$ & $*$ & $*$ & $*$ & $*$ & $*$ & $\mathrm{NS}$ & $*$ & $\mathrm{NS}$ \\
$\mathrm{Ax} \mathrm{B}$ & $\mathrm{NS}$ & $\mathrm{NS}$ & $*$ & $*$ & $*$ & $*$ & $\mathrm{NS}$ & $*$ & $\mathrm{NS}$ \\
\hline
\end{tabular}

*Means within the column followed by the same letter were not significantly different at $\mathrm{p}=0.05$ by LSD. NS $=$ Non-significant.

Table 10. Interaction effect of water regimes and $\mathrm{KClO}_{3}$ rates and on the stomatal conductance rate $\left(\mathrm{mol} \mathrm{M}^{-2} \mathrm{~S}^{-1}\right)$ after treatment

\begin{tabular}{llllllllll}
\hline \multirow{2}{*}{ Treatments } & \multicolumn{8}{c}{ Time after application (days) } \\
\cline { 2 - 10 } & 1 & 4 & 7 & 10 & 13 & 17 & 21 & 28 & 35 \\
\hline $\mathrm{WW}+\mathrm{KClO}_{3}$ & 0.03 & $0.03 \mathrm{~b}$ & $0.03 \mathrm{~b}$ & $0.03 \mathrm{~b}$ & $0.03 \mathrm{~b}$ & $0.04 \mathrm{bc}$ & 0.05 & $0.02 \mathrm{~b}$ & 0.05 \\
$\mathrm{WW}-\mathrm{KClO}_{3}$ & 0.05 & $0.07 \mathrm{a}$ & $0.07 \mathrm{a}$ & $0.07 \mathrm{a}$ & $0.07 \mathrm{a}$ & $0.07 \mathrm{a}$ & 0.06 & $0.04 \mathrm{a}$ & 0.06 \\
$\mathrm{WD}+\mathrm{KClO}_{3}$ & 0.02 & $0.02 \mathrm{~b}$ & $0.02 \mathrm{~b}$ & $0.02 \mathrm{~b}$ & $0.03 \mathrm{~b}$ & $0.03 \mathrm{c}$ & 0.05 & $0.02 \mathrm{~b}$ & 0.05 \\
$\mathrm{WD}-\mathrm{KClO}_{3}$ & 0.03 & $0.03 \mathrm{~b}$ & $0.04 \mathrm{~b}$ & $0.04 \mathrm{~b}$ & $0.04 \mathrm{~b}$ & $0.06 \mathrm{ab}$ & 0.06 & $0.02 \mathrm{~b}$ & 0.05 \\
\hline F-test & $\mathrm{NS}$ & $*$ & $*$ & $*$ & $*$ & $*$ & $\mathrm{NS}$ & $*$ & $\mathrm{NS}$ \\
\hline
\end{tabular}

*Means within the column followed by the same letter were not significantly different at $\mathrm{p}=0.05$ by LSD. NS $=$ Non-significant. 


\subsection{Changes of Volumetric Water Content and Leaf Water Potential}

The volumetric water content in the growing media after treatment showed that the water deficit reduced the moisture value while there was no effect between $10 \mathrm{~g}$ and $0 \mathrm{~g} \mathrm{KClO}_{3}$ treatment. In addition, there was no interaction among all the treatments (Table 11).

The leaf water potential after the start of a treatment showed that the well-watered had higher leaf water potential than that of water deficit at 10 to 35 days after treatment while there was no effect on that from $10 \mathrm{~g}$ and $0 \mathrm{~g} \mathrm{KClO}$. However, there was no interaction effect among treatments (Table 12).

Table 11. Effects of $\mathrm{KClO}_{3}$ and water regime on the volumetric water content of growing medium after treatments

\begin{tabular}{llllllllll}
\hline \multirow{2}{*}{ Factors } & \multicolumn{7}{c}{ Volumetric water content (\%) } \\
\cline { 2 - 10 } & 1 & 4 & 7 & 10 & 13 & 17 & 21 & 28 & 35 \\
\hline Water (A) & & & & & & & & & \\
WW & $10.76 \mathrm{a}$ & $9.80 \mathrm{a}$ & $10.41 \mathrm{a}$ & $10.30 \mathrm{a}$ & $10.53 \mathrm{a}$ & $10.28 \mathrm{a}$ & $10.08 \mathrm{a}$ & $10.00 \mathrm{a}$ & $9.93 \mathrm{a}$ \\
$\mathrm{WD}$ & $6.81 \mathrm{~b}$ & $5.83 \mathrm{~b}$ & $5.46 \mathrm{~b}$ & $5.09 \mathrm{~b}$ & $4.96 \mathrm{~b}$ & $4.39 \mathrm{~b}$ & $4.11 \mathrm{~b}$ & $4.70 \mathrm{~b}$ & $4.44 \mathrm{~b}$ \\
$\mathrm{KClO}_{3}(\mathrm{~B})$ & & & & & & & & & \\
$10 \mathrm{~g}$ & 8.70 & 7.81 & 7.69 & 7.20 & 7.36 & 6.75 & 6.38 & 6.73 & 6.53 \\
$0 \mathrm{~g}$. & 8.88 & 7.81 & 8.19 & 8.19 & 8.13 & 7.91 & 7.81 & 7.98 & 7.84 \\
\hline $\mathrm{A}$ & $*$ & $*$ & $*$ & $*$ & $*$ & $*$ & $*$ & $*$ & $*$ \\
$\mathrm{~B}$ & $\mathrm{NS}$ & $\mathrm{NS}$ & $\mathrm{NS}$ & $\mathrm{NS}$ & $\mathrm{NS}$ & $\mathrm{NS}$ & $\mathrm{NS}$ & $\mathrm{NS}$ & $\mathrm{NS}$ \\
$\mathrm{Ax} \mathrm{B}$ & $\mathrm{NS}$ & $\mathrm{NS}$ & $\mathrm{NS}$ & $\mathrm{NS}$ & $\mathrm{NS}$ & $\mathrm{NS}$ & $\mathrm{NS}$ & $\mathrm{NS}$ & $\mathrm{NS}$
\end{tabular}

*Means within the column followed by the same letter were not significantly different at $\mathrm{p}=0.05$ by LSD. NS $=$ Non-significant.

Table 12. Effects of $\mathrm{KClO}_{3}$ and water regime on the leaf water potential of longan trees after treatment

\begin{tabular}{llllllllll}
\hline \multirow{2}{*}{ Factors } & \multicolumn{8}{c}{ Leaf water potential (MPa) } \\
\cline { 2 - 10 } & 1 & 4 & 7 & 10 & 13 & 17 & 21 & 28 & 35 \\
\hline Water (A) & & & & & & & & & \\
WW & -1.22 & -1.28 & -1.25 & $-1.04 \mathrm{a}$ & $-1.02 \mathrm{a}$ & $-1.02 \mathrm{a}$ & $-0.95 \mathrm{a}$ & $-1.01 \mathrm{a}$ & $-0.92 \mathrm{a}$ \\
$\mathrm{WD}$ & -1.35 & -1.46 & -1.50 & $-1.93 \mathrm{~b}$ & $-2.21 \mathrm{~b}$ & $-2.20 \mathrm{~b}$ & $-2.35 \mathrm{~b}$ & $-2.26 \mathrm{~b}$ & $-2.28 \mathrm{~b}$ \\
$\mathrm{KClO}_{3}(\mathrm{~B})$ & & & & & & & & & \\
$10 \mathrm{~g}$ & -1.38 & -1.38 & -1.41 & -1.52 & -1.65 & -1.75 & -1.81 & -1.77 & -1.74 \\
0 g. & -1.23 & -1.35 & -1.33 & -1.45 & -1.58 & -1.47 & -1.49 & -1.50 & -1.46 \\
\hline $\mathrm{A}$ & $\mathrm{NS}$ & $\mathrm{NS}$ & $\mathrm{NS}$ & $*$ & $*$ & $*$ & $*$ & $*$ & $*$ \\
$\mathrm{~B}$ & $\mathrm{NS}$ & $\mathrm{NS}$ & $\mathrm{NS}$ & $\mathrm{NS}$ & $\mathrm{NS}$ & $\mathrm{NS}$ & $\mathrm{NS}$ & $\mathrm{NS}$ & $\mathrm{NS}$ \\
$\mathrm{Ax} \mathrm{B}$ & $\mathrm{NS}$ & $\mathrm{NS}$ & $\mathrm{NS}$ & $\mathrm{NS}$ & $\mathrm{NS}$ & $\mathrm{NS}$ & $\mathrm{NS}$ & $\mathrm{NS}$ & $\mathrm{NS}$ \\
\hline
\end{tabular}

*Means within the column followed by the same letter were not significantly different at $p=0.05$ by LSD. NS $=$ Non-significant.

\section{Discussion}

The full irrigation had faster the days of terminal bud break about 9 days. The well-watered and water deficit treatments gave approximately $43.34-47.82 \%$ of all buds flowered and $44.95-45.89 \%$ of leaf flushing. The reduction of the water amount had effect on time of terminal bud brake due to water deficiency was a factor that usually causes the limitation of growth and metabolic activity rates of the plant (Boland et al., 1993). Borchert (1994) reported that water deficit inhibited bud break and shoot growth in tropical tree. In the present study, the $91 \%$ of all buds flowered at 25-27 days after the application of $\mathrm{KClO}_{3}$. The off-season flowering in longan trees 
was induced by $\mathrm{KClO}_{3}$ (Sritontip et al., 2005; Hegele et al., 2008; Davenport \& Stern, 2005; Manochai et al., 2005). The $\mathrm{KClO}_{3}$ application induced floral emergence and reduced leaf flushing, whereas the treatments without $\mathrm{KClO}_{3}$ application did not induce flowering. The efficiency of photosystem II ( Fv/Fm), leaf net $\mathrm{CO}_{2}$ assimilation, transpiration and stomatal conductance rates were reduced in water deficit and $\mathrm{KClO}_{3}$ application, except for the application of combination of full irrigation and without $\mathrm{KClO}_{3}$ treatments. In longan trees treated with water deficit, $\mathrm{KClO}_{3}$, and a combination of water deficit and $\mathrm{KClO}_{3}$ leaf photosynthesis decreased because water deficit caused closure of the stomata and reduced $\mathrm{CO}_{2}$ assimilation and stem extension, leaf expansion, and fruit growth (Flore \& Lakso, 1989; Menzel, 2005). The high photosynthesis rate indicated the optimal irrigation management for longan and low value was led to drought stress. The photosynthetic rate of apricot trees daily irrigated to $25 \%$ of field capacity was lowered by $55 \%$ compared to control trees ( $100 \%$ field capacity), while a $75 \%$ reduction in photosynthesis was observed in the rest of water deficit stressed treatments (Ruiz-Sanchez et al., 2000). Diurnal changes in leaf gas exchange in well-water and drought were studied in Tai So litchi trees. Stomata conductance and net $\mathrm{CO}_{2}$ assimilation reached maximum values at $0700-0800 \mathrm{~h}$, and were lower in drought trees than in the controls for most of the day (Menzel \& Simpson, 1994; Menzel, 2005). Water stress decreased the net $\mathrm{CO}_{2}$ assimilate in papaya (Marler et al., 1994). Induced reduction in net $\mathrm{CO}_{2}$ assimilated and stomata conductance were also observed in Valencia orange trees (Syvertsen \& Lloyd, 1994). In Kensington mango trees effective stomatal closure was reached at -1.2 and -3.0 MPa (Schaffer et al., 1994; Pongsomboon, 1991). The water deficit had a lower leaf water potential in longan trees at 10 days after treatment due to decreasing of moisture content in growing media. The reduction of leaf water potential decreased leaf photosynthesis characteristics in longan tree because water deficit led to decreasing turgor pressure (Akinci \& Lösel, 2012). Moreover, Drought conditions are usually associated with a decrease in plant productivity and the course of growth leads to the increase of abscisic acid (ABA) and decrease of indole-3-acetic acid (IAA) and cytokinins (CKs), which may result in the early stoppage of branch growth in comparison with its natural trend (Bradford \& Hsiao, 1982; Ferguson et al., 1992)

The treatment with $\mathrm{KClO}_{3}$ induced the longan flowering process and could be used for off-season longan production. The mechanism of how $\mathrm{KClO}_{3}$ induces the flowering process in longan is not entirely understood. Some researches claimed that in plants, the chlorate $\left(\mathrm{ClO}_{3}{ }^{-}\right)$ion competitively inhibited the nitrate reductase enzyme and is reduced to chlorite $\left(\mathrm{ClO}_{2}^{-}\right)$and hypochlorite $\left(\mathrm{ClO}^{-}\right)$(Duke, 1985; King, 1974). Furthermore, the reduction products chlorite $\left(\mathrm{ClO}_{2}^{-}\right)$and hypochlorite $\left(\mathrm{ClO}^{-}\right)$were shown to be rapidly acting toxins that poisoned all plant cell types (Aberg, 1947). It was previously shown that $\mathrm{KClO}_{3}$ application also decreased chlorophyll fluorescence and leaf gas exchange (Sritontip et al., 2010), the leaf photosynthesis considerably decreased up to 6 days after $\mathrm{KClO}_{3}$ application and remained rather low compared to the control up to 11 days (Hegele et al., 2008), consequently, the detrimental effects of $\mathrm{KClO}_{3}$ on the plant's photosynthetic system could be caused by the phytotoxic effect of $\mathrm{ClO}_{2}{ }^{-}$and $\mathrm{ClO}^{-}$. Thus, water deficit and $\mathrm{KClO}_{3}$ treatments seemed to be the inhibiting factors of the photosynthetic efficiency.

Floral initiation in longan is dependent on cool temperature and some chemicals treatment. However, the leaf photosynthesis was reduced during flower induction stage by low temperature and $\mathrm{KClO}_{3}$, whereas the longan trees can induce flowering after treatments, which probably account for the depression in leaf photosynthesis rate reposed during the floral initiation in subtropical tree species (Sritontip et al., 2010; Hegele et al., 2008).There is considerable evidence for the regulatory role of plant hormones controlling floral induction, particularly in perennial fruit trees. It has been reported for trees that an increase of CKs stimulates flower induction, while high levels of gibberellic acids (GAs) and IAA result in inhibition (Bangerth, 2009). Furthermore, it was found that CKs concentrations in terminal buds of longan increased, whereas IAA concentrations reduced during the first fourteen days after $\mathrm{KClO}_{3}$ application and GAs also decreased at twenty days after treatment (Hegele et al., 2008).

Although, water deficit and $\mathrm{KClO}_{3}$ decreased leaf photosynthesis, water deficit treatment reduced media volumetric water content and leaf water potential, while both $\mathrm{KClO}_{3}$ concentrations did not significantly affect these parameters.

\section{Conclusion}

The water deficit delayed time of terminal bud break by 9 days, while, the full irrigation and water deficit treatments gave similar flowering and leaf flushing percentages. Whereas, the longan trees in treatments without $\mathrm{KClO}_{3}$ application did not have floral emergence. The efficiency of photosystem II (Fv/Fm), leaf net $\mathrm{CO}_{2}$ assimilation and transpiration rates, and stomata conductance were reduced in water deficit and with $\mathrm{KClO}_{3}$ treatments. The volumetric water content and leaf water potential declined with the water deficit treatment, while there was no difference between $10 \mathrm{~g}$ and $0 \mathrm{~g} \mathrm{KClO}_{3}$. 
Although water deficit impacted on the efficiency of photosystem II ( $\mathrm{Fv} / \mathrm{Fm})$, leaf net $\mathrm{CO}_{2}$ assimilation and transpiration rates, and stomata conductance; it did not affect off-season flower induction by $\mathrm{KClO}_{3}$. Therefore off-season production during dry season or under controlled deficit irrigation seems to be feasible, at least, in terms of how effective $\mathrm{KClO}_{3}$ is as a flower inducing agent. Irrigation management during further fruit development still requires detailed investigation in order to optimize yield and quality of off-season longan fruit.

\section{Acknowledgements}

This project has been supported by The Uplands Program (SFB 564 of the Deutsche Forschungsgemeinschaft.), Institute of Special Crops Cultivation and Crop Physiology, Faculty of Agricultural Science, University of Hohenheim, Stuttgart, Germany and Center of Excellence on Agricultural Biotechnology (Ag-BIO/PERDO-CHE), Bangkok, Thailand. We thank Dr. Rainer Zawadzki and Dr. Wirut Ampun (RMUTL) for helping with the manuscript.

\section{References}

Aberg, B. (1947). On the mechanism of the toxic action of chlorates and some related substances upon young wheat plants. Ann R Agric Cell Sweden, 15, 37-10.

Akinci, S., \& Lösel, D. M. (2012). Plant Water-Stress Response Mechanisms. In I. Md. M. Rahman, \& H. Hasegawa (Eds.), Water Stress (pp. 15-42). Rijeka, Croatia. In Tech.

Bangerth, K. F. (2009). Floral induction in mature, perennial angiosperm fruit trees: Similarities and discrepancies with annual/biennial plants and the involvement of plant hormones. Sci. Hort., 122(2), 153-163. http://dx.doi.org/10.1016/j.scienta.2009.06.014

Boland, A. M., Mitchell, P. D., Jerie, P. H., \& Goodwi, N. I. (1993). The effect of regulated deficit irrigation on three water use and growth of peach. J. Hort. Sci., 68(2), 261-279.

Borchert, R. (1994). Water status and development of tropical trees during seasonal drought. Trees, 8, 115-125.

Bradford, K. J., \& Hsiao, T. C. (1982) Physiological responses to moderate water stress. In O. L. Lange, P. S. Nobel, C. B. Osmond, \& H. Ziegle (Eds), Physiological Plant Ecology II, Encyclopedia of Plant Physiology, New Series (Vol. 12B, pp. 263-324). Berlin, Germany: Springer-Verlag.

Caspari, H. W., Behboudian, M. H., \& Chalmers, D. J. (1994). Water use, growth, and fruit yield of 'Hosui' Asian pears under deficit irrigation. J. Amer. Soc. Hort. Sci., 119, 383-388.

Chalmers, D. J., Mitchell, P. D., \& Heek, L. Van. (1981). Control of peach tree growth and productivity by regulated water supply, tree density, and summer pruning. J. Amer. Soc. Hort. Sci., 106, 307-312.

Davenport, T. L., \& Stern, R. A. (2005). Flowering. In C. M. Menzel, \& G. K. Waite (Eds.), Litchi and Longan Botany, Production and Uses (pp. 87-113). Oxfordshire, England: CABI Publishing. http://dx.doi.org/10.1079/9780851996967.0087

Dubey, R. S. (1997). Photosynthesis in Plant under Stressful Condition. In M. Pessesakli (Ed.), Handbook of Photosynthesis (pp. 859-875). New York, United State: Marcel Dekker Inc.

Duke, S. O. (1985). Effect of herbicides on nonphotosynthetic biosynthetic process. In S. O. Duke (Ed.). Weed Physiology Vol I Reproduction and Ecophysiology (pp. 91-106). Florida, CRC Press Inc, Boca Raton.

Ferguson, L., Steven, S., \& Martin, G. C. (1994). Olive Production manual. Division of Agriculture and Natural Resources, University of California, California, United State, Publication 3353.

Fitter, A. H., \& Hay, R. K. M. (1987). Environment Physiology of Plant (2nd edn). London, England: Academic Press Limited.

Flore, J. A., \& Lakso, A. N. (1989). Environment and physiological regulation of photosynthesis in fruit crops. Horticultural Reviews, 11, 111-157.

Hegele, M., Manochai, P., Naphrom, D., Sruamsiri, P., \& Wünsche, J. N. (2008). Flowering in longan (Dimocarpus longan $\mathrm{L}$.) induced by hormonal changes following $\mathrm{KClO}_{3}$ applications. Europ. J. Hort. Sci., 73, 49-54.

Hoagland, D. R., \& Arnon, D. I. (1938). The Water-Culture Method for Growing Plants Without Soil. California Agricultural Experimental Station. Circ. 347. California, United State.

King, L. J. (1974). Weeds of the World. Wiley Eastern Private Limited, New Delhi. 
LaBrie, S. T., Wilkinson., J. Q., \& Crawford, N. M. (1991). Effect of chlorate treatment on nitrate reductase and nitrate reductase gene expression in Arabidopsis thaliana. Plant Physiol., 97, 873-879. http://dx.doi.org/10.1104/pp.97.3.873

Manochai, P., Sruamsiri, P., Wiriya-Alongkone, W., Naphrom, D., Hegele, M., \& Bangerth, F. (2005). Year around off season flower induction in longan (Dimocarpus longan, Lour) trees by $\mathrm{KClO}_{3}$ applications: potentials and problems. Scientia Hort., 104, 379-390. http://dx.doi.org/10.1016/j.scienta.2005.01.004

Marler, T. E., George, A. P., Nissen, R. J., \& Andersen. P. C. (1994). Miscellaneous Tropical Fruits. In B. Schaffer, \& P. C. Andersen (Eds). The Handbook of Environmental Physiology of Fruit Crops Vol. II. Subtropical and Tropical (pp. 199-224). Boca Raton, Florida, United State, CRC Press.

Menzel, C. M. (2005). Plant Water relations and Irrigation. In Menzel C. M., \& Waite, G. K. (Eds). Litchi and Longan Botany, Production and Uses (pp. 183-207). Oxfordshire, England: CABI Publishing. http://dx.doi.org/10.1079/9780851996967.0183

Menzel, C. M., \& Simpson, D. R. (1994). Lychee. In B. Schaffer, \& P. C. Andersen (Eds), The Handbook of Environmental Physiology of Fruit Crops Vol. II. Subtropical and Tropical (pp. 123-145). Boca Raton, Florida, United State, CRC Press.

Metheneg, P. D., Ferguson, L., Goldhamer, D. A., \& Dunai, J. (1994). Effect of irrigation on Manzanilla olive flowering and shoot growth. Acta Horticulturae, 356, 168-171.

Pongsomboon,W. (1991). Effect of temperature and water stress on tree growth, flowering, fruit growth and relation of mango (Mangifera indica L.). Ph.D. Thesis, Kasetsart University, Bankok, Thailand.

Ruiz-Sanchez, M. C., Domingo, R., Torrecillas A., \& Perez-pastor, A. (2000). Water stress preconditionnng to improve drought resistance in young apricot plants. Plant Science, 156, 245-251. http://dx.doi.org/10.1016/S0168-9452(00)00262-4

Shackel, K. A., Lampinen, B., Southwick, S., Olson, W., Sibbett, S., Krueger, W., \& Yeager, J. (2000). Deficit irrigation in prunes: maintaining productivity with less water. HortScience, 35, 1063-1066.

Shaffer, B., Whiley, A. W., \& Crane, J. H. (1994). Mango. In B. Schaffer, \& P. C. Andersen (Eds.), The Handbook of Environmental Physiology of Fruit Crops Vol. II. Subtropical and Tropical (pp. 165-198). Boca Raton, Florida, United State: CRC Press.

Sritontip, C., Khaosumain, Y., Changjeraja, S., \& Poruksa, R. (2005). Effects of potassium chlorate, sodium hypochlorite and calcium hypochlorite on flowering and some physiological changes in 'Do' longan. Acta Hort. (ISHS), 665, 269-274.

Sritontip, C., Tiyayon, P., Naphrom, D., Sruamsiri, P., Manochai, P., Hegele, M., \& Wünsche, J. (2010). Effects of temperature and potassium chlorate on leaf photosynthesis and flowering in longan. Acta Hort. (ISHS), 863, 323-328.

Stecher, P. G., Finkel, M. J., Siegmund, O. H., \& Szafranski, B. M. (1960). The Merck Index of Chemicals and Drugs (7th Eds.). Rahway, New Jersey, USA: Merck \& Co, Inc.

Subhadrabandhu, S. (1990). Lychee and Longan. Faculty of Agriculture, Kasetsart University, Bangkok, Thailand.

Syvertsen, J. P., \& Lloyd, J. J. (1994). Citrus. In B. Schaffer, \& P. C. Andersen (Eds.), The Handbook of Environmental Physiology of Fruit Crops Vol. II. Subtropical and Tropical (pp. 65-100). Boca Raton, Florida, United State: CRC Press.

Thomson, W. T. (1993). Agricultural Chemicals, Book II: Herbicides. California, United State: Thomson Publications. 